\title{
Kirche und Betriebsverfassung
}

\author{
Teuteberg, Hans Jürgen
}

First published in:

Zeitschrift für Evangelische Ethik, 4. Jg., S. 27 - 45, Gütersloh 1960

Münstersches Informations- und Archivsystem multimedialer Inhalte (MIAMI) URN: urn:nbn:de:hbz:6-66459524257 


\section{Kirche und Betriebsverfassung}

von

\section{HANS JURGEN TEUTEBERG}

Die Betriebsverfassung als Ausdruck der Sozialform des Betriebes kann ohne $Z$ weifel als einer der wesentlichen Bestandteile unserer Wirtschafts- und Sozialordnung angesehen werden. Als solche steht sie in enger Verbindung mit den Ordnungsvorstellungen der Gestaltungsfaktoren der Gesamtgesellschaft, zu denen unter anderen der Staat, die Kirche und die Arbeitgeber- und Arbeitnehmerverbände zu rechnen sind. Der vorliegende Bei$\operatorname{trag}^{1}$ möchte das Beziehungsfeld Kirche und Betriebsverfassung herausgreifen und die Frage stellen: In welcher Weise hat man im evangelischen Raum bisher zur Sozialgestaltung des industriellen Betriebes Stellung genommen, und welche künftigen Probleme ergeben sich daraus für die Soziallehre und kirchliche Praxis? Wenn sich auch die evangelische Kirche grundsätzlich aus dem Streit der Tagesmeinungen heraushalten soll, so kann und darf es ihr jedoch nicht gleichgültig sein, in welcher betrieblichen Sozialordnung die ihr anvertrauten Menschen leben. Sie hat nicht nur dann zu sprechen, wenn diese Ordnung ihre religiösen und ethischen Fundamente bedroht, sie sollte auch dann Stellung beziehen, wenn es "nur« um den Menschen geht.

Bei der vielschichtigen Bedeutung der Betriebsverfassung in arbeitsrechtlicher, politischer, ökonomischer und soziologischer Hinsicht erscheint es notwendig, zunächst eine begriffliche Abgrenzung vorzunehmen und methodologische Grundsatzfragen zu erörtern. Denn die Erfahrung lehrt, daß der Meinungskonflikt zwischen den Sozialpartnern, zum Beispiel über die Grenzen der Mitbestimmung, nicht unwesentlich durch unklare Begriffsbildung gefördert wurde. Auch im kirchlichen Raum besteht hier ohne Zweifel noch eine Reihe von Unklarheiten und Fehlvorstellungen. Erst nach Feststellung gewisser Grundlinien der Betriebsverfassung soll daher auf die Bedeutung dieses Problems für die Kirche näher eingegangen werden.

Die Betriebsverfassung können wir als einen Teil der allgemeinen Sozialordnung ansehen - als die Ordnungsvorstellung vom Betrieb.

Die Einbeziehung des Betriebsgeschehens in den Zusammenhang einer größeren allgemeinen Sozialordnung entspricht der gewandelten Einstellung zum Betrieb, in diesem nicht nur eine Quelle zum Gelderwerb zu sehen. Das Geschehen in den Betrieben wird jetzt nicht mehr wie zur Zeit des wirtschaflichen Liberalismus als die privatrechtliche Angelegenheit einzelner Vertragskontrahenten, sondern mehr und mehr als gesamtgesell-

1. Es handelt sich hier um einen wenig veränderten und gekürzten Vortrag, den der Verfasser am 3. Juni 1958 vor dem Arbeitskreis »Beruf und Betrieb» des Sozialausschusses der Evangelischen Landeskirche von Westfalen in Villigst gehalten hat. 
schaftliches Phänomen verstanden. Diese Auffassung von der Betriebsverfassung als Ordnungsvorstellung vom Betrieb wird auch von evangelischer Seite zur Begriffsbestimmung herangezogen. Die Betriebsverfassung ist, so heißt es bei $M$. Donath, »die auf allgemeinen oder besonderen Gesetzen, auf Tarif-oder Betriebsvereinbarungen sowie auf tatsächlicher Ubung beruhende rechtliche und tatsächliche Ordnung von Betrieben in dem Sinne, daß das Maß der Anteilnahme der für das Betriebsgeschehen wesentlichen Faktoren an der betrieblichen Willensbildung festgelegt wird² «. Es geht also um eine angemessene Beteiligung der Arbeitnehmer an der Verwaltung der Betriebe der gewerblichen Wirtschaft. Diese Definition vertritt aber einen zu formalen Aspekt von der Betriebsverfassung, worüber an anderer Stelle noch mehr zu sagen ist.

Die Mitbestimmung ist in diesem Sinne nur eine spezielle Form der Betriebsverfassung. Juristisch ist sie das in verschiedenen Formen und Stufen in Erscheinung tretende, insbesondere im Mitbestimmungsgesetz und im vierten Teil des Betriebsverfassungsgesetzes geregelte Recht auf Mitwirkung und Mitbestimmung der Arbeitnehmer. Daneben hat sich der Ausdruck »Mitbestimmung" aber auch als Oberbegriff für alle möglichen Formen der deutschen Betriebsverfassung eingebürgert, was oftmals zu Mißverständnissen Anlaß gegeben hat. Um diesem vorzubeugen, soll die Mitbestimmung darum hier auf den eingeengten rechtlichen Bereich beschränkt bleiben und die Betriebsverfassung als Sammelbegriff für alle möglichen Stufen und Formen der Beteiligung der Arbeitnehmer an der Betriebsgewalt in sozialer, personaler und wirtschaftlicher Hinsicht verstanden werden. Der Begriff Betriebsverfassung ist fernerhin gegenüber der Wirtschaftsverfassung abzugrenzen, für deren Einzelformen oft auch die Bezeichnungen »überbetriebliche Mitbestimmung" und "Wirtschaftsdemokratie" gebraucht werden. Hier handelt es sich um die Schaffung von überbetrieblichen Bezirks- und Landeswirtschaftsräten sowie einem Bundeswirtschaftsrat, die paritätisch besetzt werden sollen. Diese Pläne um eine Wirtschaftsverfassung, für die es in der Vergangenheit einige Vorläufer gibt, sind hier nicht näher zu behandeln, zumal sie auch noch im Mittelpunkt der Auseinandersetzungen zwischen den Sozialpartnern stehen und im Gegensatz zur betrieblichen Mitbestimmung noch nicht realisiert sind. Neben der Beschränkung auf die betriebliche Ebene muß noch eine weitere thematische Abgrenzung getroffen werden. Es soll nur die Betriebsverfassung in wirtschaftlich gelenkten Unternehmen untersucht werden. Die Mitbestimmung in den öffentlichen Verwaltungsbetrieben wird heute beispielsweise durch das Personalvertretungsgesetz geregelt und bedarf daher einer eigenen Darstellung. Die von kirchlicher Seite oftmals im Zusammenhang mit der Mitbestimmung angeschnittene Frage des Miteigentums und der Gewinnbeteiligung, also die Beteiligung der Arbeiter am materiellen Betriebserfolg in Form von Arbeiteraktien, Produktivgenossenschaften, Investments usw., soll hier ebenfalls außer Betracht bleiben.

Der solchermaßen nach außen hin abgegrenzte Begriff der Betriebsverfassung trägt aber weiterhin auch noch in sich einen doppeldeutigen Charakter, was in der von M. Donath aufgestellten Definition nicht zum Ausdruck kommt. Die Verfassung der Betriebe wird einmal als institutionelle Rechtsnorm, zum anderen als bestimmter Ausdruck der inneren 2. Donath, Martin: Betriebsverfassung. In: Evangelisches Soziallexikon. Stuttgart 1954. Sp. $171 \mathrm{ff}$.
Sozialsphäre und des Betriebsklimas verstanden. Das heißt, einmal wird bei der Betriebsverfassung nach den kodifizierten Normen einer Beteiligung der Arbeitnehmer an der Verwaltung der Betriebe gefragt, wo die globalen Ordnungsvorstellungen an Hand von Gesetzen konkret nachgeprüft werden können. Zum anderen stehen die im einzelnen nicht fixierbaren menschlichen Beziehungen zwischen den Leitenden und Ausführenden im Mittelpunkt. Die Betriebsverfassung behandelt somit an Hand der rechtlichen Fakten die formale Struktur einer Beteiligung der Arbeitnehmer (äußere Verfassungsform) und zugleich die Motivationen und innere Einstellung zum Betrieb (innere Verfassungswirklichkeit).

Zwischen diesen beiden Wesenszügen der Betriebsverfassung, die im nachfolgenden noch ausführlich erläutert werden sollen, besteht eine enge Verbindung, wobei festzustellen ist, daß der Betriebsverfassung als Ausdruck der inneren Sozialsphäre der Betriebe letztlich die größere Bedeutung zukommt. Gerade die Mitbestimmung als spezielle Form der Betriebsverfassung hat gezeigt, daß sie mehr als ein Problem der guten Zusammenarbeit im Betrieb oder etwa der "Human Relations « ist. In Wahrheit geht es hier um entscheidende Grundsätze menschlichen Zusammenwirkens in der modernen Industriegesellschaft überhaupt. Der Schwerpunkt liegt nicht bei der institutionellen Form, die letztlich nur den äußeren Rahmen abgeben kann. Viel wichtiger erscheint das Wesen und der dahinter stehende Gehalt der Betriebsverfassung. Die Mitbestimmung vor allem ist nicht so sehr eine Frage der Paragraphen als vielmehr des geistigen Gehaltes, in dem sie erfüllt werden.

Es hat sich immer wieder gezeigt, daß beim Fehlen des ehrlichen Willens zur Zusammenarbeit auch das Gesetz nicht weiterhelfen konnte.

\section{Die Betriebsverfassung ALS ÄUSSERE RechtSForm}

Die deutsche Betriebsverfassung hat als Rechtsform in der Vergangenheit in einer Reihe von Gesetzen ihren Niederschlag gefunden und ist durch vielerlei Bindungen in der Sozialgeschichte verankert. Schon der Volkswirtschaftliche Ausschuß der Verfassunggebenden Nationalversammlung in Frankfurt am Main hatte 1848/49 in seinem Entwurf einer Reichsgewerbeordnung gesetzlich verankerte paritätische "Fabrikausscbüsse * vorgesehen. Die erste gesetzliche Regelung einer Beteiligung der Arbeiter am Betriebswillen der Unternehmer findet sich dann in dem Arbeiterschutzgesetz vom 1. Juni 1891. Die dort vorgesehenen "Arbeiterausschüsse ", die allerdings noch nicht obligatorisch waren, sollten vor Erlaß der Arbeitsordnung gehört werden und besaßen somit ein beschränktes Mitwirkungsrecht bei der Erfüllung sozialer Aufgaben. Die ersten gesetzlichen Betriebsvertretungen, die obligatorisch vorgeschrieben waren, wurden unter dem Druck der Kriegsereignisse mit dem Gesetz über den Vaterländischen Hilfsdienst am 5. 12. 1916 in den meisten deutschen Betrieben eingeführt. Als wesentlich neues Moment enthielt das Gesetz die Bestimmung, daß die Arbeiterausschüsse bei Arbeitsstreitigkeiten paritätisch besetzte Schlichtungsausschüsse mit einem neutralen Vorsitzenden anrufen konnten. Der Bergbau war 1900 in Bayern und 1905 in Preußen bereits mit der Einführung obliga- 
torischer Bergarbeiterausschüsse vorgezogen. Dieser hat sich, was hier von einiger Wichtigkeit ist, überhaupt als Schrittmacher auf dem Gebiet der Betriebsverfassung gezeigt. Das Betriebsrätegesetz vom 4.2. 1920 sanktionierte und erweiterte das Mitwirkungsrecht in sozialen und personellen Fragen für die Betriebsräte, wie die Arbeiterausschüsse jetzt genannt wurden. Die Unternehmer hatten die Pflicht, den Betriebsrat über wichtige Betriebsangelegenheiten zu informieren und bei betrieblichen Veränderungen mit ihm zusammenzuarbeiten; auch konnten Betriebsratsmitglieder nach einem Gesetz vom 15.2.1922 in den Aufsichtsrat entsandt werden. Mit dem Gesetz zur Ordnung der nationalen Arbeit vom 20. 1. 1934 wurde die Entwicklung der Betriebsverfassung unterbrochen. Dem Unternehmer, jetzt »Führer des Betriebes« genannt, stand ein Vertrauensrat der »Gefolgschaft « beratend zur Seite, ohne einen wirklichen Einfluß zu haben. Lediglich die überbetrieblichen und mit amtlichen Schiedsbefugnissen ausgerüsteten »Treubänder der Arbeit konnten noch in die Unternehmerbefugnisse eingreifen. Nach dem Zusammenbruch von 1945 gestattete das Kontrollratsgesetz Nr. 22 vom 10.1. 1946 wieder die Einsetzung von Betriebsräten in der alten Form. Auf Grund dieses alliierten Rahmengesetzes wurden von 1948 bis 1950 in acht von den elf westdeutschen Ländern Betriebsratsgesetze erlassen, die aber zu einer unerwünschten Rechtszersplitterung auf dem Gebiete des Arbeitsrechts führten.

Die wirklich einschneidenden gesetzlichen Umgestaltungen der deutschen Betriebsverfassung erfolgten erst durch das Gesetz über die Mitbestimmung der Arbeitnebmer in den Aufsichtsräten und Vorständen der Unternebmen des Bergbaues und der Eisen und Stabl erzeugenden Industrie vom 21. Mai 1951 mit dem Ergänzungsgesetz über die Mitbestimmung in den Holding-Gesellschaften vom 7. August 1956 sowie durch das Betriebsverfassungsgesetz vom 14. Oktober 1952. Hier haben die gesetzgeberischen Bestrebungen um die Ausgestaltung der deutschen Betriebsverfassung ihren bisher sachlichen Höhepunkt, wenn auch sicherlich nicht ihren Abschluß gefunden.

Wenn wir zunächst das Betriebsverfassungsgesetz genauer betrachten, so erkennen wir ein unterschiedliches $\mathrm{Maß}$ von Teilhaberrechten der Belegschaft an der betrieblichen Willensbildung. Es ist dies eine Staffelung der Befugnisse, die von einem einfachen Anhörrecht (Informationsrecht) über eine begrenzte Mitwirkung bis zur gleichberechtigten Mitbestimmung reichen. Bei den sozialen Angelegenbeiten kann der Betriebsrat mitbestimmen: bei der Aufstellung der Betriebsordnung, Regelung der Arbeitszeit und Pausen, bei den Fragen der Lohnregelung, bei der Unfallverhütung, bei der Verwaltung von Wohlfahrtseinrichtungen, Berufsausbildung usw.

Bei den personellen Angelegenbeiten hat der Betriebsrat in den Betrieben mit mehr als zwanzig wahlberechtigten Arbeitnehmern teils mitzubestimmen, teils mitzuwirken; nämlich bei Einstellungen, Umgruppierungen, Versetzungen, Kündigungen usw. Ein Mitbestimmungsrecht hat er bei geplanten Betriebsveränderungen, einschließlich der Einführung neuer Arbeitsmethoden. Für die Behandlung dieser wirtschaftlichen Angelegenheiten ist in Betrieben mit mehr als 100 Arbeitnehmern ein Wirtschaftsausschuß zu bilden, der aber sonst nur Anhörungs- und Beratungsrechte besitzt. Der Wirtschaftsausschuß ist nach dem Betriebsverfassungsgesetz paritätisch besetzt, während die Aufsichtsräte juristischer Per- sonen des Privatrechtes, zum Beispiel der Aktiengesellschaft, der GmbH, der Erwerbsund Wirtschaftsgenossenschaften usw., nur zu einem Drittel mit Vertretern der Arbeitnehmer beschickt werden.

Weitergehend in dieser Richtung ist das Mitbestimmungsgesetz vom 21. 5. 1951, das auf dem Gesetz der alliierten Hohen Kommission über die Umgestaltung des deutschen Kohlenbergbaues und der deutschen Stahl- und Eisenindustrie aufbaut. Hier können die Arbeitnehmer ihren vollen Einfluß auf allen oben erwähnten Gebieten geltend machen, allerdings unter Beschränkung auf bestimmte Betriebsleitungsebenen, besonders den Aufsichtsrat, in dem sie vollberechtigte Mitglieder sind. In der Regel besteht der Aufsichtsrat aus elf Mitgliedern, wobei fünf von den Anteilseignern und fünf von den Arbeitnehmern $\mathrm{zu}$ benennen sind. Bei den letztgenannten üben teils die Betriebsräte, teils die Gewerkschaften das Vorschlagsrecht aus. Der elfte Mann wird mit einfacher Stimmenmehrheit aller Stimmen der bereits gewählten Aufsichtsratsmitglieder dem Wahlorgan vorgeschlagen und von diesem - wie übrigens alle Mitglieder - gewählt. Gewerkschaften und Arbeitgeberverbände sind durch eine Reihe von Bestimmungen, die hier nicht näher erläutert zu werden brauchen, mit der heutigen Betriebsverfassung verzahnt worden. Mit ihnen kommen auch Größen zur Mitwirkung, die ihren Schwerpunkt außerhalb des Betriebes haben.

Im Bereich des Mitbestimmungsgesetzes Kohle und Eisen ist besonders auf die ohne Vorbild dastehende Institution des Arbeitsdirektors im Vorstand hinzuweisen. Rechtlich gesehen, ist er Arbeitgeber und gleichberechtigtes Vorstandsmitglied, darf aber nach dem Mitbestimmungsgesetz nicht gegen die Stimmen der Arbeitnehmervertreter im Aufsichtsrat bestellt oder abberufen werden. Er bildet eine Vertretung der Arbeitnehmer im Bereich der betrieblichen Exekutive.

\section{Die Betriebsverfassung als inNere VerfassungswirkLichKeIt}

Bei den hier skizzierten Formen der Betriebsverfassung handelt es sich um die gesetzlich verankerten Rechtsnormen. Bei ihnen ist noch nicht gesagt, inwieweit sie der tatsächlichen Ordnung in der Wirklichkeit entsprechen. Die nur an einer Rechtsordnung orientierte Betrachtungsweise der Betriebsverfassung kann in keiner Weise als ausreichend erscheinen. Denn mit der Rechtsnorm ist noch nichts über die tatsächliche Herrschaft im Betrieb, über den Betriebston, über die Arbeitsfreude und die dadurch bedingte Einstellung des Arbeiters zum Betrieb sowie über das Betriebsklima gesagt. O. Neulob hat es in seinen grundlegenden Untersuchungen über die deutsche Betriebsverfassung unternommen, sie auch unter soziologischem Aspekt zu sehen und zu deuten ${ }^{3}$. Nach ihm läßt der Ausdruck Betriebsverfassung $z$ wei Deutungsmöglichkeiten zu: einmal die »Verfaßtheit « durch Gesetze, Verträge und Vereinbarungen, zum anderen kann man damit einen Zustand bezeichnen, so wie man bei einem Menschen von seiner körperlichen, seelischen und geistigen »Verfassung « spricht. Mutatis mutandis heißt das: Welche Verfassung hat der 3. Neulob, Otto: Die deutsche Betriebsverfassung und ihre Sozialformen bis zur Mitbestimmung. Tübingen 1956. S. 33. 
Betrieb, und in welcher Verfassung befindet sich der Betrieb? Die Betriebsverfassung im soziologischen Sinne erfaßt alle die im institutionellen Bereich nicht mehr greifbaren zwischenmenschlichen Beziehungen und wird definiert als $\gg$ die von inner- und außerbetrieblichen Faktoren beeinflußte Sozialordnung eines Betriebes, für deren Gestaltung die institutionellen Formen und betriebsklimatischen Auswirkungen von entscheidender Bedeutung sind". Bei der soziologischen Betrachtungsweise läßt sich die Betriebsverfassung der letzten 150 Jahre in eine Stufenfolge bringen, die von der unbeschränkten Alleinherrschaft des Unternehmers über die freiwillig oder vertraglich beschränkten Arbeitgeberrechte bis zur gesetzlich beschränkten Betriebsgewalt reicht, in der die gewählten Arbeitnehmervertreter mehr oder minder gleichberechtigt an der Leitung der Betriebsgeschäfte teilnehmen. Für diese Rangfolge einer inneren Sozialverfassung der Betriebe gibt es natürlich zahlreiche Untergruppen und Mischformen. Als wichtig ist hier nur festzuhalten, daß die unbeschränkte Fabrikherrschaft zu Beginn der Industrialisierung und die gleichberechtigte Mitentscheidung, wie sie im Mitbestimmungsgesetz Kohle und Eisen vom 21. 5. 1951 zum Ausdruck kommt, die beiden äußersten Extreme in diesem Entwicklungsschema bilden. Bei der Skizzierung dieser Entwicklungsreihe der Betriebsverfassung wurden absichtlich die in der Literatur sonst gebräuchlichen Bezeichnungen, wie »Patriarchalismus «, »Betriebsdemokratie«, »Fabrikmonarchie», »Grubenmilitarismus«, »Herr-imHause-Standpunkt«, »konstitutionelle Fabrik « usw., nicht verwandt. Es sind dies alles Vergleiche aus der alten Familienverfassung, den Bereichen des Heeres und der staatlichen Politik, die aus Mangel an eindeutigen Begriffsbildungen zur Beschreibung dieser neuartigen Phänomene des Betriebes dienen mußten. Im Grunde bedeuten sie eine Übertragung betriebsfremder Vorstellungen. Ebenso wie die Verhältnisse eines Familienstiles (Vater-Eigenschaft des Unternehmers, Vormund der Arbeiter usw.) für den modernen Betrieb als irreal empfunden werden, ebenso unzweckmäßig erscheint auch die Gleichsetzung mit dem Stil einer parlamentarischen Demokratie. Selbst die eifrigsten Verfechter einer Demokratisierung der Betriebe haben inzwischen eingesehen, daß eine Werksleitung nicht von dem Willen einer Belegschaft in parlamentarischer Weise abhängig gemacht werden kann. Die Leitung eines Betriebes vollzieht sich in einer völlig anderen Weise, wie denn für Staat und Wirtschaft völlig eigene Gesetze gelten, die sich nicht ohne weiteres übertragen lassen. Damit soll keineswegs bestritten werden, daß diese vergleichenden Vorstellungen in der Sozialgeschichte eine außerordentlich große Rolle gespielt haben. Es geht unseres Erachtens aber nicht an, wenn gerade im kirchlichen Raum immer noch mit diesen inadäquaten Leitbildern, insbesondere aus dem Familien- oder Gemeindeleben, operiert wird. Auch Begriffe, wie zum Beispiel Betriebsgemeinschaft, die durch die Wirtschaftsfriedliche Bewegung und das Dritte Reich in ihrem ursprünglichen Sinngehalt entwertet wurden, sollten nach den Erkenntnissen der Betriebssoziologie besser nicht mehr verwandt werden.

Bei dieser Gelegenheit muß darauf hingewiesen werden, daß es in der Vergangenheit nicht an Unternehmern fehlte, die, ihrem sozialen Gewissen folgend, freiwillig Herrschaftsvorrechte an die gewählte Betriebsvertretung abtraten und zusammen mit den Forderungen der Sozialreformer dadurch die gesetzliche Regelung entscheidend beeinflußten. Die
Vorläufer der deutschen Mitbestimmung 4 gehen mit ihren Anfängen weit in die deutsche Sozialgeschichte zurück. Aus ihrer Entwicklung läßt sich ablesen, daß der Gedanke eine Beteiligung der Arbeiter an den Unternehmerbefugnissen im Grunde so alt ist wie das Fabrikwesen überhaupt in Deutschland. Zahlreiche Quellenzeugnisse des 19. Jahrhundert zeigen, in welcher Weise einzelne Unternehmer zum Schrittmacher eines weiteren Aus baues der Betriebsverfassung wurden, während die organisierte Arbeiterschaft erst nach Abwendung von der sozialrevolutionären Kampfansage im Revisionismus und dem Aufkommen der Gewerkschaften positiven Einfluß auf die Entwicklung nahm. Dem aus Unkenntnis der Dinge aufgestellten Vorurteil, der Gedanke an eine soziale Betriebsverfassung sei ein Produlst der Jahre nach dem ersten und zweiten Weltkrieg und eine reine Gewerkschaftsforderung, muß mit Nachdruck entgegengetreten werden.

II.

Nach Skizzierung dieser Grundlinien der Betriebsverfassung ist die Frage zu stellen, welche Stellung die evangelische Kirche zu dem Problem einer Betriebsverfassung bisher eingenommen hat und wie sie heute dazu steht. In zahlreichen Darstellungen ist beschrieben worden ${ }^{5}$, wie die seit der Reformation bestehende Bindung zwischen "Thron und Altar und der seit der Confessio Augustana geforderte prinzipielle Gehorsam gegenüber der weltlichen Obrigkeit die Auseinandersetzung der Kirche mit den Problemen der industriellen Arbeitswelt erschwert haben. Die evangelische Kirche stand der Industrialisierung, die die Menschen aus jahrhundertealten und traditionalen Bindungen löste, mit tiefer Skepsis und Sorge gegenüber. Besonders das neue Maschinenwesen stieß bei ihr fast auf vollständige Ablehnung. Die kürzlich veröffentlichten Memoiren eines evangelischen Arbeitersekretärs ${ }^{6}$ aus dem Württembergischen zeigen noch einmal mit seltener Deutlichkeit, wie sehr die lärmenden Fabriksäle von der Mehrzahl der Geistlichen als Störungsfaktor der gewohnten patriarchalischen Ordnung empfunden wurden, wie ängstlich es vermieden wurde, an den neuen Herd der sozialen Spannungen vorzudringen, wie sehr man sich im Hinterhaus der wilhelminischen Gesellschaft auf das rein Missionarische beschränkte. Auch die Amsterdamer Kirchenkonferenz von 1948 unterstrich, daß »die Kirchen nicht vergessen (sollten), in welchem Ausmaß sie selbst gerade zu den Übeln beigetragen haben, die sie so gern der Verweltlichung der Gesellschaft zur Last legen ... Unsere Kirchen haben oft die besonderen Vorrechte der herrschenden Klassen, Rassen und politischen Gruppen religiös sanktioniert und sind auf diese Weise den Wandlungen im Wege gewesen, die im Interesse der sozialen Gerechtigkeit und politischen Freiheit notwendig waren ${ }^{7}$.

4. Sie wurden vom Verfasser zum Gegenstand einer Untersuchung gemacht: Hans Jürgen Teuteberg: Vorläufer der deutschen Mitbestimmung. Diss. phil. Göttingen 1957 (Maschinenschrift).

5. Vgl, unter anderem Ernst Troeltsch: Die Soziallehren der christlichen Kirchen und Gruppen. 3. Aufl. Tübingen 1923. S. 585 ff.; Emil Brunner: Das Gebot und die Ordnungen. 1932 (nachgedruckt von der amerikanischen Hilfskommission des Ökumenischen Rates der Kirchen). S. 310 ff.; Eckart Scbletb: Der profane Weltchrist. Neubau der Lebensform des Industriemenschen. München 1957.

6. Springer, August: Der Andere das bist Du. Tübingen 1954.

7. Amsterdamer Dokumente, hg. von Focko Lüpsen, Bethel b. Bielefeld, 2. Aufl., S. 47. 
Von E. Scbleth wird mit Recht betont, daß die ersten sozialen Programme auf proteter 作 tischen Versuche von Johann Friedrich Oberlin, Gustav Werner, Karl Mez und Johann . Wichern erwarteten die Lösung der sozialen Spannungen von einer christlichen Erneuerung der Arbeiter und der »sittlichen Regeneration der Fabrik ${ }$. Die von diesen Männern geleistete karitative Arbeit war aus dem Gedanken einer konservativ geprägten ing für die gewandelte gesellschaftliche Strate karitative Arbeit rüttelte zwar die Bench notig waren. Die karitaive Arbeit rütelte zwar die Kirche aus ihrer Passivität gegenüber weltlichen Dingen auf, doch war sie im Grund genommen nur ein Rückgriff auf die Methoden der alten patriarchalischen »Armenpolizey ". Der in Armut darbende Mensch war der Gesuchte, nicht aber der Proletarier, der . eine neue Ordnung hat $^{9}$. Wieviel in das Gedankengut der evangelischen Kirche in übernommen wurde, zeigen die christlich-sozialen Bemühungen des Berliner Hof prediger Adolf Stöcker, des märkischen Pastors Rudolf Todt, des hannoverschen Kirchenleiters Gustav Ublborn und des aus W/uppertal stammenden Greifswalder Theologen Martin von Nathasius. Erst Victor Aimé Huber, der Verfechter der Selbsthilfe in Form der Produktivenossenschaten, Friedrich Naumann mit seinen Gedanken über die Industrieverfassung, die praktischen Versuche des Berliner Jalousie-Fabrikanten Heinrich Freese sowie die auf die staatliche Sozialpolitik einwirkenden Ideen des Bismarck-Beraters Theodor Lobmann und das Programm des Evangelisch-Sozialen Kongresses brachten einen erten Durchen des damaligen konservativen protestantischen Denkens ${ }^{10}$.

Der Theologe Ernst Troeltsch vollzog schließlich im Bezirk der systematischen Theologie dann der Industriegesellschaft bejahte.

Diese Bemühungen sind zugleich ein Beweis dafür, daß die Kirche bzw die führenden Dersönlichkeiten und Gruppen nicht achtlos an diesem Teil der sozialen Frage vorbeigegangen sind. Sie zeigen aber auch die Unzulänglichkeiten der liberalen Wir können uns in diesem Rahen auf die Nennung dieser wenigen Namen mit dem Hinweis darauf beschränken, wieviel Verbindungslinien noch heute zu diesem

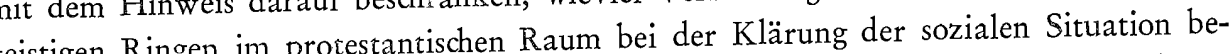

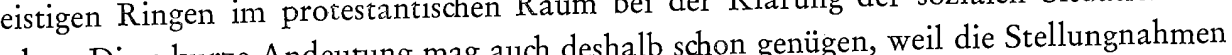
theologie von damals

-

8. Vg1. Paul Wurster: Gustav Werners Leben und Wirken. Reutlingen 1888, Jobannes Kober Kalte Schrifein Vorkämpfer für christlichen Sozialismus. Basel 1892

ten, hg. von Friedrich

9. Schleth, a.a.O., S. 28.

10. Huber, Victor Aimé: Ausgewählte Schritten über Sozialreform und Genoss Fralfurt am Main 1949; Friedrich Naumann: Ausgewählte Schriften, hg. von Max Graf zu Soln. That Heinrich Freese: Die konstitutionelle Fabrik. Jena 1909; Hans Robbfels: Theolor Lohm Kampfjahre der staatlichen Sozialpolitik. Berlin 1927. anknüpfen. Der Wandel im theologischen Denken, der vor allem mit dem Namen Karl Barth verbunden ist, wurde besonders auf dem 1. Evangelischen Kirchentag in Bethel 1924 und der Weltkirchenkonferenz für praktisches Christentum in Stockbolm 1925 sichtbar. Hier kam die durch die Erschütterungen des ersten Weltkrieges veranlaßte Besinnung auf das eigentliche Wesen des Glaubens und der christlichen Botschaft zum Ausdruck. Auf diese Besinnung folgte eine Hinwendung zu den Fragen, die sich aus dem Verhältnis zur Welt stellten, und damit eine Bereitschaft der Kirche, aus den überkommenen gesellschaftlichen Denkvorstellungen herauszukommen. Die Frage nach einer neuen glaubwürdigen Sozialordnung und damit auch nach dem Problem der Betriebsverfassung wurde in ein ganz neues Licht gestellt.

Der Zusammenbruch von 1918 hatte die alte Symbiose » Thron und Altar « weitgehend zerschlagen und die evangelische Kirche frei gemacht, sich ohne prinzipielle Vorurteile mit ihrer sozialen Verantwortung zu beschäftigen. Was am Ende des 19. Jahrhunderts die Angelegenheit einiger weniger überzeugter kirchlicher Kreise war, reifte jetzt im großen heran, da die Kirchentage immerhin halbkirchenamtlichen Charakter hatten. In Bethel hieß es, Unternehmer und Arbeiter seien »zu gemeiner Arbeit Berufene und aufeinander Angewiesene «. In Stockholm wurde schon konkreter gesagt: »Der Organisation der Industrie soll als Ziel der Entwicklung ... die Zusammenarbeit aller Beteiligten vorschweben. Dadurch wird bei den Arbeitern das Bewußtsein des Wertes ihrer Arbeit erhöht, und sie werden fühlen, daß sie nicht bloß ein Spielball fremder Interessen sind ... es scheint gerecht, daß sie auch an der Leitung (Control) einen gewissen Anteil haben wollen, besonders da, wo es sich um ihre eigensten Interessen handelt und wo sie im Stande sind, etwas Wirksames zur Förderung der Produktion beizutragen ${ }^{11}$. Es ist ein besonderer Zug dieser Außerungen, daß sie einen weltweiten ökumenischen Aspekt tragen. Die Erklärungen der Weltkirchenkonferenzen, wie sie auch nach dem zweiten Weltkrieg in Amsterdam $^{12}$ und Evanston abgehalten wurden, mußten aber die unterschiedlichen Sozial- und Wirtschaftsstrukturen der dort vertretenen zahlreichen Länder berücksichtigen. Ein Eingehen auf spezielle Probleme der Einzelstaaten verbot sich von selbst. Der ökumenische Aspekt führte dazu, daß die Erklärungen nur allgemein gehalten werden konnten und keine konkreten Einzelvorschläge für die Umgestaltung der Betriebe enthalten. Ein wichtiger Kristallisationspunkt für die evangelische Sozialarbeit zwischen 1918 und 1933 bildete auch die unter dem Einfluß Friedrich Brunstäds gegründete Evangelisch-Soziale Schule in Berlin-Spandau, an deren Wirken alle späteren evangelischen Akademien im Grunde wieder anknüpften. Schon um diese Zeit hat es eine Reihe von Sozialpfarrern gegeben, die im Zuge der Auseinandersetzung mit dem Werksgemeinschaftsgedanken der Wirtschaftsfriedlichen Bewegung dem Problem einer sozialen Betriebsverfassung nähertraten, wie zum Beispiel das Referat Wilhelm Menns auf dem 34. Evangelisch-sozialen

11. Vgl. Verhandlungen des Ersten Deutschen Evangelischen Kirchentages, ho. vom Evangelischen Preßverband für Deutschland. Berlin-Steglitz 1924; Adolf Keller: Soziale Programme der Kirche und freien
balsher religiösen Organisationen. Zürich 1929; Heinrich Kraut: Kirche und Mitbestimmung. Bielefeld 1950. S. 12; Schleth, a.a.O., S. $77 \mathrm{ff}$.

12. Amsterdamer Olsumenisches Gespräch, Bd. 3. Furche Verlag, Tübingen, und Evangelisches Verlagswerk, Stuttgart 1948; Kraut, a.a.O., S. 13.

34 
Kongreß 1927 in Hamburg zeigt ${ }^{13}$. Wie nach dem ersten Weltkrieg ist auch nach 1945 eine neue Hinwendung der Kirche zu den Fragen der Sozialordnung und Betriebsgestaltung zu verzeichnen gewesen. Die Vernichtungswelle der großen Kriegskatastrophe riß mit allem, was sie begrub, auch viele in der Tradition verhaftete Vorurteile der Kirche gegen soziale Fragen, zugleich aber auch viele Ressentiments der modernen Arbeitswelt gegen die Kirche fort. Der Sozialethische Ausschuß der Evangelischen Kirche im Rheinland unter Friedrich Karrenberg, die Evangelische Akademie in Bad Ball unter Eberbard Müller und das Sozialamt der Evangelischen Landeskirche von Westfalen unter Klaus von Bismarck waren die ersten, die die Bemühungen der evangelischen Sozialarbeit um eine neue Betriebsgestaltung wieder aufnahmen. In den folgenden Jahren wurden 14 evangelische Akademien gegründet, die sich auf zahlreichen Tagungen mit allen sozialen Schichten um diese Fragen bemühten. Hervorzuheben sind auch die 1950 begonnene "Gemeinsame Sozialarbeit der Konfessionen im Bergbau « und die kirchliche Betriebsarbeit in der Textilindustrie. Der Zusammenschluß aller kirchlichen Verbände und Werke der EKD zu einer »Aktionsgemeinschaft für Arbeiterfragen im Jahre 1949 sowie die Errichtung der zentralen sozialen Bildungsstätte Friedewald, die in direkter Fortsetzung der Arbeit im Berliner Johannesstift entstand, waren weitere organisatorische Maßnahmen, um eine Stellungnahme der evangelischen Kirche zu diesen brennenden Sozialproblemen zu erarbeiten.

Eberhard Müller hat noch vor Erlaß des Betriebsverfassungsgesetzes und des Mitbestimmungsgesetzes als Ergebnis eines Studienkreises bereits 1950 mit seiner Schrift $\gg$ Recht und Gerechtigkeit in der Mitbestimmung ${ }^{14_{*}}$ einen evangelischen Ratschlag zu geben versucht. Darin stellte er die Notwendigkeit einer neuen betrieblichen Sozialordnung und ihre gesetzliche Sicherung mit Nachdruck heraus. Wenn heute Mitbestimmungsrechte der Arbeiter gefordert werden, sagte er, so ist dies zu einem wesentlichen Teil die Folge davon, daß in dem vergangenen Jahrhundert, in dem die heutige Wirtschaftsform entstanden ist, die Aufgabe der Mitbestimmung nicht in genügender Weise erkannt wurde. Auch die aus christlicher und sittlicher Pflicht handelnden Unternehmer hätten in der Vergangenheit zu oft übersehen, daß für einen dauerhaften Frieden in der Wirtschaft eine gerechte Entlohnung und freiwillige Sozialfürsorge allein nicht ausreichen, ihre Mitarbeiter von dem drückenden Gefühl der Abhängigkeit zu befreien. Zur Befriedigung am gemeinsamen Werk gehöre auch die Teilnahme an der Verantwortung. Die christlichen Arbeitnehmer hätten ihrerseits übersehen, daß zu einer echten Mitbestimmung nicht nur der Kampf um materielle Rechte gehöre, sondern auch die Bereitschaft, wirtschaftliche Verantwortung für das gemeinsame Werk zu tragen.

Müller vergleicht den Betrieb von heute mit einer sportlichen Team-Mannschaft, die den größten Erfolg erst im rechten Zusammenspiel erhalten könne. Trotz aller Gesetze und freiwilligen Vereinbarungen hat sich dieses Zusammenspiel aber nur selten erreichen

13. Auf die Tätigkeit der Berliner Evangelisch-sozialen Schule machte mich freundlicherweise Prof. Schweitzer aufmerksam. Zur Rede Wilhelm Menns vgl. Schleth, a.a.O., S. 76.

14. Müller, Eberhard: Recht und Gerechtigkeit in der Mitbestimmung. In: Schriftenreihe "Der Deutschenspiegel«, Bd. 36/37, Stuttgart 1950. lassen. Der Verfasser erkennt durchaus die Schwierigkeiten bei der Herstellung eines solchen Vertrauensverhältnisses und fordert, das Verhältnis der Beteiligten dürfe auf keinen Fall so geordnet werden, daß einer den anderen in seinen Rechten benachteilige oder in seinen ihm zugehörenden Verantwortungsbereichen blockieren könne. Sonst könne leicht anstatt des allgemeinen Vertrauens ein Krieg aller gegen alle mit Anwendung sämtlicher zur Verfügung stehender finanzieller, rechtlicher und politischer Druckmittel die nicht verwischen. Denn, wenn die Gesetzgebung die Befugnisse so ineinandermischt, daß keiner mehr ohne den anderen $z u$ irgendeiner Handlung fähig ist, so erreicht man kein Vertrauen, sondern eine Art von gesetzlicher Willkür überbetrieblicher Instanzen des stärkeren und rücksichtsloseren Sozialpartners.

Diese erste größere Stellungnahme zur Mitbestimmung im evangelischen Raum bekenn sich ferner eindeutig zum Recht auf das persönliche Eigentum. $Z_{\text {war sei die Gemeinschaft }}$ der Menschen verpflichtet, die Eigentumsrechte überall dort $\mathrm{zu}$ begrenzen, wo sie in ungerechter Weise und zum Schaden anderer verwendet werden, sie kann insbesondere unrechtmäßig erworbene oder dem Allgemeinwohl schädliche Eigentumsrechte durch gesetzliche Maßnahmen aufheben oder einschränken, aber sie ist zugleich verpflichtet, darauf zu achten, daß hier nicht die Besitzgier einzelner Machthaber oder politischer Mehrheiten ungleiches Recht unter den Bürgern errichtet. Damit wird von evangelischer Seite ganz klar ausgesprochen, daß der Gesetzgeber die bestehenden Eigentumsverhältnisse durch neue ersetzen muß, wenn er deren Rechtmäßigkeit anzweifelt, er soll es aber vermeiden, sie - ohne sich für eine Änderung bewußt zu entscheiden - im Mitbestimmungsrecht vorwegzunehmen.

Diese Stellungnahme kennzeichnet zugleich, wo nach evangelischer Auffassung die Grenzen einer Mitbestimmung liegen müssen.

Ähnliche Gedankengänge kehren in der Erklärung des Rates der EKD zur Mitbestimmung auf dem Essener Kirchentag 1950 wieder ${ }^{15}$, die auf Beratungen der Sozialkammer der EKD zurüdkgeht. Der Sinn des Mitbestimmungsrechtes sei es, so heißt es dort, das bloße Lohnarbeitsverhältnis zu überwinden und den Arbeitnehmer als Menschen und Mitarbeiter ernst zu nehmen. Dabei würden freilich aus der Sache heraus Abstufungen zwischen sozialen, betriebstechnischen, persönlichen und wirtschaftlichen Mitbestimmungsrechten unerläßlich sein. Besonderes Gewicht wird auf ein organisches Hineinwachsen der Beteiligten in die Verantwortung und auf Spielraum für freie Vereinbarung gelegt. Der Platz der Verbände soll vor allen Dingen in der überbetrieblichen Mitbestimmung sein. Bei der Gestaltung des betrieblichen Mitbestimmungsrechtes müsse der Tatsache Rechnung getragen werden, $d a ß$ in erster Linie die Angehörigen des Betriebes selbst zur Mitverantwortung berufen sind. Damit wird von evangelischer Seite eine institutionelle Neuordnung der Betriebsverfassung bejaht. Das Schlußmemorandum der ersten Arbeitsgruppe des

15. Abdruck in: Schriftenreihe Kirche und Volk, H. 6 (1950), S. 60. Vg1. auch Evangelisches Soziallexikon, a.a.O., Sp. 729. - Auch die Arbeitsgruppe "Wirtschate auf dem 1. Kirchentag 1949 in Hannover wie auch alle späteren Kirchentage haben sich in besonderer Weise diesen Dingen zugewandt. Vgl. Martin Donath: Die soziale Frage auf dem Evangelischen Kirchentag. In: Evangelisches Soziallexikon, a.a.O., Sp. 594 
Essener Kirchentages geht aber auch auf die dahinterstehende innere Sozialverfassung der Betriebe ein. Es heißt dort, man solle dieBedeutung des Institutionellen nicht überschätzen. Der Bestand oder das Scheitern rechtlicher Institutionen auf diesem Gebiet sei von dem Geist abhängig, der in ihnen lebendig ist, dem Geist des Friedens und der Versöhnung oder dem Geist des Haders und der Entzweiung. Diese Erklärung des Rates der EKD auf dem Essener Kirchentag ist bis heute die wichtigste offiziöse Stellungnahme auf evangelischer Seite zur Betriebsverfassung geblieben.

Die führenden evangelischen Sozialpolitiker waren sich aber auch bewußt, daß dieses nur eine allgemeine Wegweisung sein konnte. Um die vielschichtige und komplexe Materie näher zu durchleuchten, trat auf Anregung des Sozialamtes der Evangelischen Kirche Westfalens Anfang 1950 der Villigster Studienkreis "Verantwortliche Gesellschaft « zusammen, um im Verein mit allgemein gesellschattichen Problemen und anderen Fragen der christlichen Verantwortung auch das Problem der Betriebsverfassung erneut anzugehen. Der unter der Leitung von Alfred Müller-Armack stehende Studienkreis, dem neben Theologen auch Vertreter der Wirtschaftswissenschaften und Soziologie sowie Männer der sozialen und wirtschaftlichen Praxis angehörten, nahm dann in seinem Abschlußbericht auch ausführlich zu den Aufgaben des Betriebes in der industriellen Gesellschaft Stellung16. Darin werden als allgemeine Grundgedanken herausgestellt:

Der Betrieb ist nicht nur eine technische und kaufmännische Einheit, sondern auch eine Personengemeinschaft. Daraus ergibt sich, daß weder Kapital, noch Technik, noch Organisation die Alleinverfügung über die im Betrieb tätigen Menschen erhalten können, sondern in eine ihrem Wesen und ihrer Funktion entsprechende Ordnung zu den Menschen und ihren Gruppenbildungen gebracht werden müssen. Aus den Erfordernissen des technischen Betriebsvollzuges, aus seiner Abhängigkeit vom Markte und seinen produk-

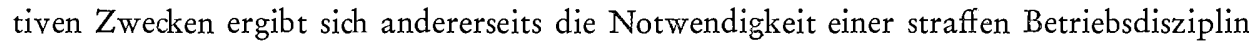
und klaren Führungsverantwortung. Die Funktion des Betriebes, insbesondere des Großbetriebes, als Existenzraum der in ihm Tätigen und ihrer Familien einerseits und als Teile des Versorgungsraumes der Bevölkerung mit den Gütern des täglichen Bedarfs andererseits, gibt ihm eine öffentliche Verantwortung, die neben den privatwirtschaftlichen Charakter eine sozialwirtschaftliche Verpflichtung stellt. Aus diesen wichtigen Grundgedanken werden dann für die zwischenmenschlichen Beziehungen im Betrieb und eine Beteiligung der Arbeitnehmer an den Unternehmerrechten einige Schlußfolgerungen gezogen.

Der moderne Industriebetrieb hat sich längst aus den alten sozialen Verpflichtungen und traditionellen Bindungen gelöst, die früher für den Handwerkerhaushalt bestanden. Seine Funktionen sind so arbeitsteilig geworden, daß er den Einzelmenschen nicht mehr in seiner Ganzheit zu umfassen vermag. Der Arbeiter sieht sich menschlicher Unzulänglichkeit und der Unsicherheit wechselnder Konjunkturen ausgesetzt: also einer anonymen Fremábestimmung, gegen die er sich nicht zu wehren vermag. Die ständige Bedrohung seiner Existenz hat zu einem tiefen Mißtrauen zur allgemeinen Sozialordnung der mensch-

16. Die heutige Gesellschaft nach evangelischem Verständnis, hg. vom Villigster Studienkreis "Verantwortliche Gesellsdraft«, Villigst 1952, S. $24 \mathrm{ff}$ lichen Gesellschaft geführt. Wir erkennen heute, daß die organisatorische Ausgestaltung der Betriebe im Hinblick auf die zwischenmenschlichen Beziehungen nicht Schritt gehalten hat. Zu ihrer Konsolidierung bedarf die industrielle Gesellschaft der Zustimmung der in ihr Arbeitenden zur Gesamtordnung und der Schaffung eines neuen sozialen Bewußtseins. Die Verbundenheit des Menschen mit der sozialen Gesamtordnung hat früher einmal bestanden. Da es jedoch unmöglich erscheint, zu den überlieferten Formen zurückzukehren, müssen neue Wege gesucht werden. Eine dem Menschen glaubwürdige Sozialordnung kann nicht allein durch den Appell an den guten Willen und den Gesinnungswechsel erreicht werden. Es bedarf daher der institutionellen Sicherung, um der Bereitschaft zur menschlichen Zusammenarbeit auch rechtlich Dauer zu verleihen. Die Institutionen, durch die die Ernsthaftigkeit des Gesinnungswechsels bezeugt wird, sind aber nur eine Stütze der Sozialordnung. Es ist von vornherein ausgeschlossen, eine bestimmte Institution als die allein mögliche anzusprechen. Als Aufgabe muß es angesehen werden, eine sinnvolle Beteiligung aller im Betrieb Arbeitenden durch die Schaffung eines gestuften Systems von Eigenverantwortlichkeiten zu ermöglichen. Sicherlich sind die komplizierten Zusammenhänge einer modernen arbeitsteiligen Wirtschaft für den einzelnen nur sehr schwer durchschaubar. Deshalb sollte nur alle im überschaubaren Tätigkeitsbereich zu tragende Verantwortung dem dort Tätigen überlassen bleiben. Für zentrale Entscheidungen soll jedoch eine Zusammenfassung der Verantwortung in Anspruch genommen werden. Unter diesem Gesichtspunkt wird von dem Villigster Studienkreis die Mitbestimmung im Betrieb gesehen. Unabhängig von den Beweggründen, die für die Einführung des Mitbestimmungsrechtes oder seine Ablehnung maßgebend waren, geht es seiner Meinung nach darum, die Möglichkeiten anzuerkennen und zu nutzen, die in dieser organisatorischen Form für die Gestaltung einer echten Partnerschaft liegen. Nach evangelischem Verständnis steht fest, daß derartige Formen nicht an sich gut oder schlecht sind, sondern nur nach Maßgabe der Gesinnung, die sich in ihnen bestätigt.

Die solcherart auf Kirchentagen und in evangelischen Sozialgremien erarbeiteten richtungweisenden Erkenntnisse haben in den letzten Jahren - wie die kleine Bibliographie im Anhang zeigt - eine Fülle von kleinen und großen Publikationen ausgelöst, von denen die von Heinrich von der Gablentz, Friedrich Karrenberg, Ludwig Heyde, Gerhard Heilfurth und Eckart Schleth sowie die betreffenden Artikel im Evangelischen Soziallexikon vor allem wohl hervorzuheben sind ${ }^{17}$. Aus Raumgründen verbietet es sich leider, sich mit ihnen allen näher zu beschäftigen.

17. Gablentz, Heinrich von der: Das Mitbestimmungsrecht der Arbeiter und Angestellten als Aufgabe der Wirtschaftspolitilk und Volksbildung. In Veritas, Justitia, Libertas, 1953. - Karrenberg, Friedrich: Mitbestimmung in der Wirtschaft. In: Kirche in Volk, H. 9. Mülheim-Ruhr 1953. - Heyde, Ludwig Abriß der Sozialpolitik. 10. Aufl. Heidelberg 1953. - Heilfurth, Gerbard: Was sollen Christen im Betrieb? (Berichte der Arbeitsgruppe IV des Hamburger Kirchentages,) In: Kirche im Volk Mülheim-Ruhr 1954. Ders.: Kirche und Arbeiterschaft. In: Friedewalder Beiträge zur Volk, H. 10 H. 5. Berlin 1954. Ders.: Zehn Grundregeln für die Zusammenarbeit. In: Die Mitarbeit, Frage, (Mai-Juni 1952). 
III.

Wenn wir die halbamtlichen Stellungnahmen der EKD und die Außerungen führender evangelischer Persönlichkeiten und Gruppen zu dem Problem der sozialen Betriebsverfassung überschauen, von denen wir hier verständlicherweise nur einige uns typisch erscheinende zitieren konnten, können wir einige Erkenntnisse daraus ziehen. Rein äußerlich ist eine weitgehende Übereinstimmung mit der katholischen Auffassung über Sinn, Stufung und Gefahren der Mitbestimmung unverkennbar, wie sie beispielsweise in der Erklärung des Bochumer Katholikentages von 1949 zum Ausdruck gekommen ist ${ }^{18}$. Wie diese zeigt sich die evangelische Auffassung in Soziallehre und Sozialpraxis weit mehr als in der Vergangenheit an einer menschlich sauberen Ordnung und einer auf gegenseitige Achtung beruhenden Zusammenarbeit im Betrieb interessiert. Ahnlich wie auf katholischer Seite wird grundsätzlich an dem Recht auf persönliches Eigentum festgehalten und durch die Mitbestimmung lediglich eine »soziale Bindung des Eigentums« unterstïtzt. Die Grundpositionen der katholischen Sozialordnung, wie sie zum Beispiel in dem Subsidiaritätsprinzip umrissen worden sind, sind bekanntlich in den beiden päpstlichen Enzykliken "Rerum novarum « (1891) und "Quadragesimo anno" (1931) niedergelegt worden. Auch die Erklärung des Bochumer Katholikentages von 1949 wurzelt nach Auffassung mancher ganz in diesen Prinzipien.

Auf evangelischer Seite wird im Gegensatz dazu auf eine philosophische Grundlegung, wie etwa nach dem Naturrecht, bewußt verzichtet. Das evangelische Verständnis wird nicht aus unverrückbaren obersten Prinzipien abgeleitet, sondern unmittelbar der Glaubenslehre entnommen. Die evangelische Kirche ist im Grunde voller Zweifel, man könnte in der Wirklichkeit bereits vorgegebene Ordnungen entdecken. Dieser bewußte Verzicht auf eine philosophische Grundlegung, die dem Wesen evangelischer Sozialethik entspricht ${ }^{19}$, bedeutet in unserem Falle: Die Kirche nimmt kritisch zu den bestehenden Sozialordnungen der Betriebe Stellung, verzichtet aber auf fertige Lösungen, um den Verantwortlichen der beiden Sozialpartner einen möglichst breiten Raum für ihr gestaltendes Handeln zu lassen. Ganz klar wird dieses ausgesprochen, wenn es in den Empfehlungen des Villigster Studienkreises zur Frage der Mitbestimmung heißt $\mathrm{t}^{20}$, es könne »nicht die Aufgabe der Kirche sein, über die wirtschaftlichen und soziologischen Techniken solcher Maßnahmen $\mathrm{zu}$ entscheiden. Notwendig ist jedoch in der heutigen Zeitlage, auf das Erfordernis einer gesicherten und glaubwürdigen Sozialordnung hinzuweisen und $z u$ jenen Versuchen $z u$ ermutigen, ja geradezu sie zur Pflicht zu machen, die gegenwärtig zur Lösung dieses Problems vorgeschlagen werden «. Hier spiegelt sich eine echte Bereitschaft zur Mitarbeit der Kirche an diesen Fragen wider, zugleich aber auch ein Mißtrauen gegen allzu perfektionistische Lösungen; eine Auffassung, die von Friedrich Karrenberg an anderer Stelle

18. Gerechtigkeit schafft Frieden. Bericht über den 73. Deutschen Katholikentag in Bochum. Bochum 1949, Paderborn 1949.

19. Auf das theologische Methodenproblem hierzu kann nicht weiter eingegangen werden. Vgl. Brunner, a.a.O., S. 380; ferner: Wirtschaft und evangelische Sozialethik. In: Fachberichte der Gesellschaft für soziale Betriebspraxis, Jg. 1956 (Novemberheft), S. 7.

20. Die heutige Gesellschaft nach evangelischem Verständnis, a.a.O., S. 28. bestätigt wird, wenn er schreibt: "Es gibt doch keinen Perfektionismus der sozialen Formen. Sie sind alle von Menschen gemacht und werden von ihnen gehandhabt. Und in diesem Punkt haben wir doch eben nicht viel Grund zu fröhlichem Optimismus ${ }^{21}$.«

Auch bei Anerkennung dieser grundsätzlichen Position der evangelischen Kirche, daß es nicht ihre Aufgabe ist, die Probleme im Detail zu entscheiden, sondern sich auf eine Wegweisung und Hilfestellung für eine glaubwürdige Sozialordnung zu beschränken, wird man doch sagen dürfen, daß die bisherigen Stellungnahmen zur Frage der Betriebsverfassung noch eine ausgeprägte Ordnungsvorstellung vom Betrieb vermissen lassen. Vier Fragen sind es, die unseres Erachtens noch nicht genügend beantwortet scheinen:

1. Wie steht die evangelische Kirche grundsätzlich zur Notwendigkeit einer Neuordnung des Betriebes?

2. Welche Grundgedanken sollen nach evangelischem Verständnis dieser Neuordnung mitgegeben werden?

3. Wie stellt sich die Kirche eine künftige Sozialgestaltung des Betriebes konkret vor? 4. Welchen Einfluß soll die Kirche auf die Realisierung solcher Ordnungsvorstellungen ausüben?

Es scheint, als ob die Bedeutung dieser Fragen noch nicht in einem genügend großen Kreis gesehen worden ist. Ein Vergleich der bisher erschienenen Literatur auf katholischer und evangelischer Seite zur Frage der Betriebsverfassung macht die hier aufgestellte Forderung deutlich. Die verstreuten Beiträge von evangelischer Seite, die manchmal hinsichtlich der Betriebsverfassung noch eine klare Begriffsbildung und Systematik vermissen lassen und häufig mit den ganz anderen Fragen des Miteigentums verquickt sind, bedürfen einer sorgfältigen Zusammenfassung. Diese müßte die allgemeinen Erklärungen und Empfehlungen des Rates der EKD auf dem Essener Kirchentag 1950 und des Villigster Studienkreises "Verantwortliche Gesellschaft « vertiefen und präzisieren sowie die von einzelnen neueren Autoren aufgezeigten Linien bis in die Gegenwart verlängern.

Es fehlt noch weitgehend an einer Konfrontation der Mitbestimmung mit der heutigen Betriebswirklichkeit nach evangelischer Sicht. Dieses erscheint aber um so notwendiger, als auch nach evangelischem Verständnis die eigentliche Arbeit an der Mitbestimmung erst nach dem Erlaß des äußeren normativen Rahmens beginnt. Eine solche Gegenüberstellung des Mitbestimmungsrechtes mit der heutigen Wirklichkeit hat den Gegenstand eingehender betriebssoziologischer Untersuchungen der Sozialforschungsstelle an der Universität Münster in Dortmund gebildet ${ }^{22}$. Befragungen auf allen hierarchischen Ebenen in einem repräsentativen Hüttenwerk des Ruhrgebietes haben gezeigt, daß die Mitbestimmung sich zu einem wesentlichen Faktor für ein neues Betriebsbewußtsein entwickelt hat. Die paritätische Besetzung des Aufsichtsrates hat sich als ein Motor für die Realisierung des sozialen Friedens im Betrieb erwiesen. Entgegen vielen Erwartungen gibt es in den Aufsichtsräten fast keine Kampfabstimmung, und die Beschlüsse sind trotz der verschie21. Karrenberg, Friedricb: Neoliberalismus - Neosozialismus. In: Gestalt und Kritik des Westens. Stutt-
gart 1959.

22. Vgl. Otto Neulob: Der neue Betriebsstil. Untersuchungen über Wirklichkeit und Wirkungen der Mitbestimmung, mit Beiträgen von Prof. Dr. Friedrich Baerwald und Dr. Markus Kühn. Tübingen 
denen Interessengruppen meistens einstimmig. Die paritätischen Aufsichtsräte haben sich vielfach zu Schulen gegenseitiger 'Toleranz entwickelt, bei der die Arbeitnehmervertreter marktwirtschaftliches, die Unternehmervertreter aber sozialwirtschaftliches Denken verstehen lernen. Die gesetzliche Pflicht zur Verschwiegenheit und der ständige persönliche Kontakt der Sozialpartner haben manche alte Gegensätze zumindest entschärft. Gleichzeitig ist eine stärkere Dezentralisation und Stärkung der unteren Verantwortungsbereiche zu beobachten gewesen. Auch die erstmals geschaffene Funktion des Arbeitsdirektors und die neuen Befugnisse der Betriebsvertretung, die sich kaum noch mit dem Betriebsrat Weimarer Prägung vergleichen lassen, können als progressive Stilwandlung des Betriebsgeschebens gedeutet werden. Schließlich ist durch die Mitbestimmung auch ein neues betriebliches Ordnungsdenken, wie es sich im allgemeinen Betriebston, der vertikalen und horizontalen Kommunikation, der Betriebsdisziplin und der Selbst- und Gruppenerziehung äußert, erkennbar geworden. An die Stelle der funktionalen, nur von oben gestützten Autorität tritt mehr und mehr die echte und natürliche Autorität. Die Herrschaft der Gewalt und des Herr-im-Hause-Standpunktes kommt bei den Belegschaften immer weniger an. Der durch die Mitbestimmung ausgeübte $Z$ wang, vieles gemeinsam zu entscheiden, hat im Vorstand ebenso wie im Aufsichtsrat zu neuen persönlichen Kontakten geführt, die früher in der Großindustrie nicht für möglich gehalten wurden.

Wir konnten hier nur einige wenige Wirkungen der Mitbestimmung auf die betriebliche Praxis andeuten. Sie zeigen jedoch schon, wie wichtig es ist, diese Probleme im kirchlichen Raum neu zu durchdenken und sich um eine Gesamtkonzeption zu bemühen. Wenn wir abschließend die Frage stellen, welchen konkreten Beitrag die Kirche bei der Erarbeitung eines grundsätzlichen Ordnungsbildes für die Schaffung eines neuen Betriebsbewußtseins leisten kann, so kristallisiert sich eine Reihe wichtiger Aufgaben heraus. Gerade weil die evangelische Sozialethik nicht an bereits vorgegebene natürliche Ordnung der Gesellschaft und Wirtschaft glaubt, sollte sie sich um so ernsthafter der wissenschaftlichen Analyse bei ihrer Wegweisung bedienen. Das bedeutet, daß sich die angesprochenen Glieder der Kirche, insbesondere die in der täglichen Sozialarbeit Tätigen, einen umfassenden Einblick in die Funktionszusammenhänge der Betriebe als Kernpunkte der Sozialordnung verschaffen soliten. Bei der Mitbestimmung hat man beispielsweise bisher häufig zu sehr nur den $» N a ̈ c h s t e n ~ i m ~ B e t r i e b »$ isoliert betrachtet. Man hat zu sehr das Individuum im Betrieb im Auge gehabt und nicht den Betrieb als einheitliches Ganzes und Bereich des Gruppenlebens in einer bestimmten Ordnung. Wenn die Kirche aber eine wirkliche Hilfestellung bei der Schaffung eines neuen Betriebsstiles leisten will, muß sie auch tiefer in das industrielle Geflecht mit seiner komplizierten Arbeitsteilung und seinen Führungsmechanismen eindringen. "Es muß endgültig die wilhelminische Auffassung vom industriellen Arbeitsverhältnis weichen, die den Unternehmer als Kapitän auf der Kommandobrücke und den Arbeiter als armen abhängigen Kohlentrimmer sieht« (K. v. Bismarck). Der Blick muß frei werden für das funktionelle Verflochtensein eines modernen Betriebes unter der heutigen Betriebsverfassung. Das bedeutet nichts anderes, als daß sich die Kirche in umfassender Weise mit allen Fakten und Möglichkeiten der Betriebsverfassung vertraut macht, womit sowohl die institutionelle äußere Rechtsnorm wie auch die tatsächliche innere Ver- fassungswirklichkeit und Sozialsphäre gemeint ist. Es muß ganz klar werden, daß zur Behandlung des Problemkomplexes Kirche und Betriebsverfassung ein gewisses Instrumentarium rechtlicher und soziologischer Art notwendig ist. In praxi heißt das die Aufnahme betriebssoziologischer und arbeitswissenschaftlicher Vorlesungen und Seminare in den Lehrplan der evangelischen Akademien und Ausbildungsstätten. Nur so ist es möglich, die noch vorhandenen Fehlurteile zu beseitigen, die meistens den Blick für die Interdependenz des wirschaftlichen und sozialen Handelns vermissen lassen.

Aber die lehrmäßige Veranschaulichung der konkreten Fakten von der heutigen Verfassung unserer Betriebe allein genügt noch nicht. Die passive Aufnahme der Wissenstatsachen bedarf noch einer Erweiterung durch ein echtes Verständnis für den Betrieb als Krafteld der sozialen Willensbildung. Es muß die Frage diskutiert werden: Wie können die Erfahrungen für das Gemeindeleben nutzbar gemacht werden? Wie kann die Diskussion darüber in den kirchlichen Unterricht, die Jugendarbeit und überhaupt in die Verkündigung hineingetragen werden? Die aufgearbeiteten Erkenntnisse und Einsichten, wie die Kirche zu der Verfassung der Betriebe steht, dürfen nicht auf die mit Sonderaufgaben betrauten kleinen Kreise wie bisher beschränkt bleiben. Es muß vielmehr in das allgemeine kirchliche Bewußtsein dringen, daß der Industriebetrieb nicht mehr als Störungsfaktor der missionarischen Arbeit, sondern als gesellschaftspolitischer Ordnungsfaktor erkannt wird. Wenn auch die Gemeindepfarrer um die Möglichkeiten und Grenzen der heutigen betrieblichen Sozialverfassung wissen, wird es der Kirche insgesamt leichter sein, an die Entstehungsherde sozialer Spannungen vorzudringen. Die in der Vergangenheit bei der evangelischen Kirche zutage getretene Verkennung des Betriebes für die Aufrichtung einer glaubwürdigen Sozialordnung soll nun nicht - auch davor muß gewarnt werden im rückläufigen Pendelschlag zu einer totalen Überbewertung des Betriebes führen. Wenn früher gewohnheitsgemäß kirchlicherseits vor der Brandmauer der Fabrik haltgemacht wurde, so soll nun der Betrieb nicht zu dem Prisma werden, in dem sich der gesamte moderne Mensch mit allen seinen Lebensäußerungen widerspiegelt. Das Ziel ist es vielmehr, den Blick ganz frei zu machen, in welcher Weise der Betrieb und seine Verfassung als Störungs- oder Ordnungsfaktor auftreten kann.

Als letzte und schwierigste Aufgabe bleibt es schließlich, eine eigene soziale Ordnungsvorstellung vom Betrieb zu entwickeln, was zugleich eine Fortbildung der evangelischen Sozialethik bedeuten würde. Bei den Dingen um die Betriebsverfassung handelt es sich längst nicht mehr um die »Arbeiterfrage « des 19. Jahrhunderts. Die Schichten im Betrieb sind viel diffuser, zahlreicher und unübersichtlicher geworden, so daß das überlieferte Zweiklassedenken von »Oben« und »Unten « zur Erfassung der sozialen Tatbestände im Betrieb nicht mehr ausreicht. An ihre Stelle müßte eine an der modernen Betriebssoziologie orientierte Gruppenetbik treten. An dieser Stelle können wir hier nur einige primäre Anregungen und Hinweise geben, die man von anderer Seite weiterführen sollte. Eine solche grundlegende Konzeption über einen neuen Betriebsstil nach evangelischer Auffassung bedeutet aber sicherlich keine Einmischung in irgendwelche Augenblicksinteressen und keine Aufgabe der grundsätzlich zurückhaltenden Position. Die Kirche könnte damit der Gesellschaft einen Dienst tun, der vielleicht von ihr am besten geleistet werden 
kann: mitzuhelfen am Abbau des gegenseitigen Mißtrauens der Sozialpartner und beim Aufbau einer Sozialordnung, mit der sich alle Arbeitenden wieder verbunden fühlen.

\section{BIBLIOGRAPHIE: EVANGELISCHE KIRCHE UND MITBESTIMMUNC}

Bismarck, Klaus v.: Kirche und moderne Arbeitswelt. In: Kirche im Volk, H. 16 (1957), 2. Aufl. Ders.: Die Freiheit des Christen zum Halten und Hergeben - Neue Welt durch Technik. Referate von Wolfgang Schwabe, C. J. Heckmann und Rudolf Müller-Schwefe auf dem Leipziger Kirchentag 1954. In: Kirche im Volk, H. 14 (1954). - Ders.: Der Betrieb als Mitarbeitergemeinschaft. Rede anläßlich der Entgegennahme des Freiherr-vom-Stein-Preises am 21. 6. 1954 in Hamburg. Abgedruckt in einer Gedenkschrift der Stiftung Freiherr vom Stein zu Hamburg, »Freiherr-vomStein-Preis 1954«. - Bobne, R.: In Dschungel der Anonymen. Gespräche in Hermannsburg. In: Die Welt, Nr. 260 v. 6. 11. 1950. - Brïder bei der Arbeit. Vorträge und Bericht der vierten Arbeitsgruppe des Berliner Kirchentages 1951. In: Kirche im Volk, H. 5 (1951). - Delekat, F.: Kirche über den Zeiten. Düsseldorf 1953. - Der Mensch im Kollektiv. Vorträge und Bericht der ersten Arbeitsgruppe des Essener Kirchentages 1950. In: Kirche im Volk, hg. von der Evangelischen Kirche im Rheinland, H. 6 (1952), Mülheim-Ruhr. - Der Wille zum gegenseitigen Versteben. Dr. Agartz und Generaldirektor Friedrich bei der Evangelischen Akademie. In: Frankfurter Allgemeine Zeitung, Nr. 276 v. 28. 11. 1950. - Die beutige Gesellschaft nach evangelischem Verständnis. Diagnose und Vorschläge zu ihrer Gestaltung. Hg. vom Villigster Studienkreis "Verantwortliche Gesellschaft«, Villigst 1952. - Donath, Martin: Kritische Tage um das Mitbestimmungsrecht. In: Junge Kirche, Oldenburg, Jg. 12 (1951), S. 58-65. - Ders.: Betriebsverfassung. In: Evangelisches Soziallexikon, Stuttgart 1954, Sp. 171 f. - Ders.: Die soziale Frage auf dem Evangelischen Kirchentag. In: Evangelisches Soziallexikon, Stuttgart 1954, Sp. 594 f. - Es ging um die Mitbestimmung. In: Welt der Arbeit, Nr. 14 v. 7. 4. 1950 (Tagung der Evangelischen Akademie in Königswinter). - Espelkamp 1955: Die Kirche und die Welt der industriellen Arbeit. Reden und Entschließungen der Synode der Evangelischen Kirche in Deutschland. Hg. von Klaus v. Bismarck. Witten (Ruhr) 1955. - Evangelische Kirche und Mitbestimmungsrecht. In: Der Betrieb, Jg. 2 (1949), Nr. 44, S. 527. - Evangelische Kirche und Mitbestimmungsrecht. In: Der Betrieb, Jg. 2 (1949), Nr. 49, S. 588. - Evangelische Akademie, Hermannsburg: Tage der Besinnung für Arbeitnehmer der Betriebe im Rheinland und Westfalen vom 28. 11. bis 2. 12.1949 und Tage der Besinnung für leitende Männer der Betriebe im Rheinland und Westfalen vom 2. bis 4. 12 1949 in Königswinter. Aussprachen, Niederschrift, getr. Pag. (Maschinenschrift). - Evangelische Kirche und Mitbestimmung. In: Industriekurier, Nr. 135 v. 2. 9. 1950. - Evanston Dokumente. Berichte und Reden auf der Weltkirchenkonferenz in Evanston 1954. Hg. von Focko Lüpsen. Witten (Ruhr) 1954. - Fischer, Rolf: Wem gehört der Betrieb? In: Kirche im Volk, H. 7 (1952). Gablentz, Heinrich von der: Das Mitbestimmungsrecht der Arbeiter und Angestellten als Aufgabe der Wirtschaftspolitik und Volksbildung. In: Veritas, Justitia, Libertas (1953). - Geissler, Wilfried: Bejahung des Mitbestimmungsrechtes. In: Der Bürger im Staat, Stuttgart, Jg. 1 (1951), H. 6, S. 85-88. - Grosse, Franz: Protestantismus und Mitbestimmungsrecht. In: Gewerkschaftliche Monatshefte, Jg. 1 (1950), H. 8, S. 345-352. - Hartmann, Paul: Die evangelische Arbeiterschaft zur Frage des Mitbestimmungsrechts im Betrieb. In: Junge Kirche, Oldenburg, Jg. 10 (1949), Sp. 619-622. - Hartung, Karlgustav: Zum Gespräch der Partner. In: Der Mensch in der Wirtschaft. Informationen aus der Arbeit der Freunde der Evangelischen Akademie, Hermannsburg, Jg. 1951, H. 1, S. 9-11. - Heilfurth, Gerbard: Was sollen Christen im Betrieb? In: Kirche im Volk, H. 10, 1954 (Berichte der Arbeitsgruppe IV des Hamburger Kirchentages). - Ders.: Kirche und Arbeiterschaft. In: Friedewalder Beiträge zur sozialen Frage, H. 5, Berlin 1954, S. 49 f. Ders.: Auf das Miteinander kommt es an. In: Die Mitarbeit, H. 1 (April 1952), S. 1. - Ders. Heinz-Mohr, Gerd: Mitwissen, Mitdenken, Mitwirken. Gedanken zu den Lehrgängen der Evangelischen Akademie Loccum für junge Menschen aus der Industrie. In: Betriebswirtschaftliche
Forschung und Praxis, Wolfenbüttel, Jg. 5 (1953), H. 6, S. 370-373. - Hessing, Erich: Mensch und Arbeit in evangelischer Sicht. In: Mensch und Arbeit, Jg. 1 (1949), H. 5, S. 102. - Heyde, Ludreig Abriß der Sozialpolitik. 10. Aufl. Heidelberg 1953. - Jacobs, Paul: Keiner ohne den anderen. In Kirche im Volk, H. 5.(1952). - Karrenberg, Friedrich: Das, Kiul: Keiner ohne den anderen. In Sozialethik. Essen 1949. - Ders. Mitbestimmung in der Wirtaft Mulhein-Ruhr 1953 (Schrefter reihe »Kirche im Volk «, H 9). Ders. Versuchung und Veran worthe Kirche im Volt . Kirche im Volk, H. 11 (1954). - Ders.: Wem gehört der Betrieb? Bericht über die Verhandlungen der 4. Arbeitsgruppe des Stuttgarter. Kirchentages 1952. In: Sozialer Fortschritt, Jg. 1 (1952), H. 10, S. 247. - Krant, Heinricb: Kirche und Mitbestimmungrecht ausschusses der Evang. Kirche in Westfalen. Bielefeld 1950. $29 \mathrm{~S}$. Kreyssig: We des SozialBetrieb? In: Kirche im Volk, H. 7 (1952) - Krimm gart 1953 - Leo, Walther. Kirche lä2). - Krimm, H.: Das diakonische Amt der Kirche. Stuttgart 1953. - Leo, Walther: Kirche kämpt gegen die Angst. Evangelische Akademie Hermannsburg lud Wirtschaftler ein. In: Neue Zeitung, Nr. 29 v. 3. 2. 1951. - Lillich, Henry: Vom Proletarier zum Mitarbeiter. In: Die Mitarbeit, Herborn, Jg. 2 (1953), H. 2, S. 1-5. - Müller, Eberbard: Kirche und Mitbestimmungsrecht. In: Stuttgarter Narion Recht und Gerechtigkeit in der Mitbestimmug. Ein evarbard: Evangelischen Akademie (Bad Boll). Stuttgr. Deutschn Deutschenspiegel«, Schriften zur Erkenntnis und Erneuerung, Bd. 36/37.) - Ders.: Diagnose: Kreislaufstörungen. In: Sozialer Fortschritt (1952), H. 3, S. 49-52. - Neue Gemeinsamkeit. In: Neue Zeitung, Nr. 203 v. 28. 8. 1950. - Radlinger, Michael: Der Arbeiter und Die Mitarbeit, Herborn, Jg. 2 (1953), H. 2, S. 5-8. - Sanden, Gerbard: Gottes Ebenbild im Großbetrieb. Offene Worte in Hermannsburg. In: Die Welt, Nr. 181 v. 31. 10. 1949. - Seeling, Otto: Wem gehört der Betrieb? In: Kirche im Volk, H. 7 (1952). - Soziale Etbik. In: Industriekurier, Nr. 106 v. 13. 9. 1949. - Scbleth, Eckbart: Der profane Weltchrist. Neubau der Lebensform für den Industriemenschen. München 1957. - Ders.: Christliche Grundsätze einer Kölner Diplomarbeit 1953. - Scblösser, H.: Mitbestimmung in zeitung, Essen, Nr. 11 v. 12 1. 1950. - Teuteberg Hans-Jügen: Voliu Sicht. In: Neue Ruhrbestimmung. Ursprung und in der Wirklichkeit und Wenken und in der Wirklichkeit des 19. Jahrhunderts (1785-1916). Diss. phil. Göttingen 1957. - Thielicke, Helmuth: Die evangelische Kirche und die Politik. Stuttgart 1953. - Tradition der Aussprache in Hermannsburg. Tagung der Evangelischen Akademie - Die Aufgaben nach Einigung der Sozialpartner. In: Handelsblatt, Nr. 10 v. 24. 1. 1951. - Wem gebört der Betrieb? In: Industriekurier, Nr. 87 v. 10. 6. 1952. - Wendland, Heinz-Dietrich: Die Kirche in der modernen Gesellschaft. Hamburg 1956. - Ders.: Partnerschaft - christlich gesehen. In. Ger modernen Gesellschaft. Jg. 6, H. 11 (November 1955). - Wendt, Siegfried: Die industrielle Unternehmung als soziales Gebilde. In: Der Mensch und die Wirtschaft, H. 4, Essen 1951. - Wirtschaft und als soziales Sozialetbik. In: Fachberichte der Gesellschaft für soziale Betriebspraxis (Nov. 1956), S. 6 ff. Zusammenarbeit im Betrieb unter christlicher Verantwortung. 1952 (Schriftenreihe der Evangelischen Akademie Hamburg, H. 5).

Dr.H.J.Teuteberg

Dortmund, Rheinlanddamm 199 